\title{
THREE-DIMENSIONAL OCTONION WAVELET TRANSFORM
}

\author{
Andrzej Katunin \\ Institute of Fundamentals of Machinery Design, Silesian University of Technology \\ Gliwice, Poland \\ andrzej.katunin@polsl.pl
}

\begin{abstract}
The necessities of processing of spatial data require developing new feature-sensitive tools such as extensions of the wavelet transform. Considering the advantages of the application of complex wavelets and their extension to quaternionic wavelets for twodimensional data structures, the new octonion discrete wavelet transform for the analysis of three-dimensional data structures was introduced in this paper. The construction of the wavelet pyramid as well as octonionic wavelets were presented.
\end{abstract}

Keywords: octonion wavelet transform, spatial data processing, octonionic wavelets

\section{Introduction}

The modern demands of spatial data processing require efficient tools for data analysis and feature extraction. One of the intensively developed branches of tools for data processing is the wavelet transform, which allows for a great variation of scaling and wavelet functions, which could be applied during the transform in order to fulfill the requirements of the specific tasks.

However, despite the great localization properties in both spatial and frequency domain, the real-valued wavelets lack the shift-invariance and directional selectivity properties. Such properties have the complex-valued wavelets [1], because the independent filterbanks of real and imaginary wavelets constitute the Hilbert transform pair [2]. This explains why great attention is paid to the complex wavelets, complex wavelet transforms and their generalizations.

Many authors developed the complex extensions of real-valued wavelets [3, 4] and new complex wavelets $[5,6]$. The natural extension of the complex wavelets and complex wavelet transform is the hypercomplex transform with appropriate wavelets. The next generalization of a complex wavelet transform is the discrete quaternion wavelet transform (QWT), which operates on the quaternion (hypercomplex) numbers of 4D vector space. The QWT was introduced in [7] and extended in [8] for the application to 2D image processing. The continuous version of QWT was introduced in [9]. Further, several researchers used QWT for various problems: Soulard and Carré [10] used QWT for texture classification, Liu et al. [2] presented a study of measuring of image sharpness based on quaternion wavelets, while the authors of $[11,12]$ applied QWT for texture and banknote classification. 
The goal of the following study is to extend the QWT algorithm applicable to 2D datasets to octonion wavelet transform (OWT), which could be applied for the analysis of 3D datasets and ensures the shift-invariance and directional selectivity properties. The formulation of OWT including the multiresolution pyramid as well as formulation of octonionic wavelets structure were under consideration in this paper.

\section{Octonion wavelet transform}

\subsection{Octonion algebra}

The octonions (known also as Cayley numbers) are the hypercomplex numbers of 8-dimensional vector space, which could be presented as:

$$
\mathbf{O}=\left\{o=x_{o}+e_{1} x_{1}+e_{2} x_{2}+e_{3} x_{3}+e_{4} x_{4}+e_{5} x_{5}+e_{6} x_{6}+e_{7} x_{7}\right\},
$$

where $\mathbf{O}$ is the octonion algebra, $x_{i} \in \mathbf{R}$ are the real coefficients and $e_{i}$ are the orthogonal imaginary numbers, i.e. $e_{i}^{2}=-1$. Considering the fact that octonions are neither commutative

$$
e_{i} e_{j}=-e_{j} e_{i} \neq e_{j} e_{i} \text { for } i, j \neq 0
$$

nor associative

$$
\left(e_{i} e_{j}\right) e_{k}=-e_{i}\left(e_{j} e_{k}\right) \neq e_{i}\left(e_{j} e_{k}\right) \text { for } i, j, k \neq 0,
$$

the multiplicative rules for them differed with respect to the complex algebra $\mathbf{C}$ or quaternionic algebra $\mathbf{H}$. The description of the operations on octonions could be found in [13]. The conjugate of $o$ is defined as:

$$
\left\{o^{*}=x_{o}-e_{1} x_{1}-e_{2} x_{2}-e_{3} x_{3}-e_{4} x_{4}-e_{5} x_{5}-e_{6} x_{6}-e_{7} x_{7}\right\},
$$

while the norm is defined as:

$$
\|o\|=\sqrt{O^{*} \cdot O}
$$

which satisfies the following property: $\left\|o_{1} o_{2}\right\|=\left\|o_{1}\right\| \cdot\left\|o_{2}\right\|$.

Extending the polar decomposition of $\mathbf{H}$ [12], the octonion $o$ could be decomposed in the same manner: $\overline{\mathbf{O}}=\mathbf{O}-\{0\} \cong \mathbf{S}^{7} \times \mathbf{R}^{+}$, where $\mathbf{S}^{7}$ is not a group as for quaternions, $\mathbf{S}^{3}=S U(2)$, it has a structure called a loop [14]. Similarly as in [12], the octonion could be decomposed as follows:

$$
o=|o| \exp \left(e_{1} \alpha\right) \exp \left(e_{2} \beta\right) \exp \left(e_{3} \gamma\right) \exp \left(e_{4} \delta\right) \exp \left(e_{5} \varepsilon\right) \exp \left(e_{6} \zeta\right) \exp \left(e_{7} \eta\right),
$$

where $|o|$ is the modulus of $o$ and $(\alpha, \beta, \gamma, \delta, \varepsilon, \zeta, \eta)$ are the phase angles. 


\subsection{Octonionic wavelet pyramid}

Following Mallat's multiresolution algorithm the 3D data structure $f(x, y, z)$ could be presented in the decomposed form as follows:

$$
\begin{aligned}
& f(x, y, z)=A_{n}^{o} f+ \\
& +\sum_{i=1}^{n}\left(D_{i, 1}^{o} f+D_{i, 2}^{o} f+D_{i, 3}^{o} f+D_{i, 4}^{o} f+D_{i, 5}^{o} f+D_{i, 6}^{o} f+D_{i, 7}^{o} f\right)
\end{aligned}
$$

where $o$ in the superscript was used for indicating octonion 3D analytic signal defined e.g. in [15], $A_{n}^{o} f(x, y, z)$ and $D_{i, j}^{o} f(x, y, z), j=1, \ldots, 7$, are the approximation and details sets of coefficients of $f(x, y, z)$, respectively. The two latter sets could be then presented in terms of scaling function $\boldsymbol{\Phi}^{o}(x, y, z)$ and wavelet functions $\boldsymbol{\Psi}_{j}^{o}(x, y, z)$ as follows

$$
\begin{gathered}
A_{i}^{o} f(x, y, z)=\sum_{k=-\infty}^{\infty} \sum_{l=-\infty}^{\infty} \sum_{m=-\infty}^{\infty} a_{i, k, l, m} \boldsymbol{\Phi}_{i, k, l, m}^{o}(x, y, z), \\
D_{i, j}^{o} f(x, y, z)=\sum_{k=-\infty}^{\infty} \sum_{l=-\infty}^{\infty} \sum_{m=-\infty}^{\infty} d_{i, j, k, l, m} \boldsymbol{\Psi}_{i, j, k, l, m}^{o}(x, y, z),
\end{gathered}
$$

where

$$
\begin{array}{r}
\boldsymbol{\Phi}_{i, k, l, m}^{o}(x, y, z)=2^{-i} \boldsymbol{\Phi}^{o}\left[2^{-i}(x-k), 2^{-i}(y-l), 2^{-i}(z-m)\right], \\
\boldsymbol{\Psi}_{i, j, k, l, m}^{o}(x, y, z)=2^{-i} \boldsymbol{\Psi}_{j}^{o}\left[2^{-i}(x-k), 2^{-i}(y-l), 2^{-i}(z-m)\right],
\end{array}
$$

and

$$
\begin{aligned}
a_{i, k, l, m} & =\left\langle f(x, y, z), \boldsymbol{\Phi}_{i, k, l, m}^{o}(x, y, z)\right\rangle, \\
d_{i, j, k, l, m} & =\left\langle f(x, y, z), \boldsymbol{\Psi}_{i, j, k, l, m}^{o}(x, y, z)\right\rangle,
\end{aligned}
$$

which could be obtained by the iterative determination of impulse responses $h^{o}$ and $g^{o}$ of the filters $\boldsymbol{\Phi}^{o}(x, y, z)$ and $\boldsymbol{\Psi}_{j}^{o}(x, y, z)$ following Mallat's algorithm.

In the case, when $\boldsymbol{\Phi}^{o}(x, y, z)$ and $\boldsymbol{\Psi}_{j}^{o}(x, y, z)$ are separable, they could be decomposed in the following way:

$$
\begin{array}{ll}
\boldsymbol{\Phi}^{o}(x, y, z)=\phi(x) \phi(y) \phi(z), & \boldsymbol{\Psi}_{4}^{o}(x, y, z)=\psi(x) \psi(y) \phi(z), \\
\boldsymbol{\Psi}_{1}^{o}(x, y, z)=\phi(x) \phi(y) \psi(z), & \boldsymbol{\Psi}_{5}^{o}(x, y, z)=\psi(x) \phi(y) \psi(z), \\
\boldsymbol{\Psi}_{2}^{o}(x, y, z)=\phi(x) \psi(y) \phi(z), & \boldsymbol{\Psi}_{6}^{o}(x, y, z)=\phi(x) \psi(y) \psi(z), \\
\boldsymbol{\Psi}_{3}^{o}(x, y, z)=\psi(x) \phi(y) \phi(z), & \boldsymbol{\Psi}_{7}^{o}(x, y, z)=\psi(x) \psi(y) \psi(z),
\end{array}
$$


where $\phi(\bullet)$ and $\psi(\bullet)$ are the 1D complex filters applied along the directions $x, y$ and $z$. Considering the filter representation of OWT the octonionic scaling and wavelet functions (14) could be presented in the following way:

$$
\begin{aligned}
& \boldsymbol{\Phi}^{o}(x, y, z)=\phi_{h}(x) \phi_{h}(y) \phi_{h}(z)+e_{1} \phi_{h}(x) \phi_{h}(y) \phi_{g}(z)+ \\
& e_{2} \phi_{h}(x) \phi_{g}(y) \phi_{h}(z)+e_{3} \phi_{h}(x) \phi_{g}(y) \phi_{g}(z)+e_{4} \phi_{g}(x) \phi_{h}(y) \phi_{h}(z)+ \\
& +e_{5} \phi_{g}(x) \phi_{h}(y) \phi_{g}(z)+e_{6} \phi_{g}(x) \phi_{g}(y) \phi_{h}(z)+e_{7} \phi_{g}(x) \phi_{g}(y) \phi_{g}(z) \text {, } \\
& \boldsymbol{\Psi}_{1}^{o}(x, y, z)=\phi_{h}(x) \phi_{h}(y) \psi_{h}(z)+e_{1} \phi_{h}(x) \phi_{h}(y) \psi_{g}(z)+ \\
& +e_{2} \phi_{h}(x) \phi_{g}(y) \psi_{h}(z)+e_{3} \phi_{h}(x) \phi_{g}(y) \psi_{g}(z)+e_{4} \phi_{g}(x) \phi_{h}(y) \psi_{h}(z)+ \\
& +e_{5} \phi_{g}(x) \phi_{h}(y) \psi_{g}(z)+e_{6} \phi_{g}(x) \phi_{g}(y) \psi_{h}(z)+e_{7} \phi_{g}(x) \phi_{g}(y) \psi_{g}(z), \\
& \boldsymbol{\Psi}_{2}^{o}(x, y, z)=\phi_{h}(x) \psi_{h}(y) \phi_{h}(z)+e_{1} \phi_{h}(x) \psi_{h}(y) \phi_{g}(z)+ \\
& +e_{2} \phi_{h}(x) \psi_{g}(y) \phi_{h}(z)+e_{3} \phi_{h}(x) \psi_{g}(y) \phi_{g}(z)+e_{4} \phi_{g}(x) \psi_{h}(y) \phi_{h}(z)+ \\
& +e_{5} \phi_{g}(x) \psi_{h}(y) \phi_{g}(z)+e_{6} \phi_{g}(x) \psi_{g}(y) \phi_{h}(z)+e_{7} \phi_{g}(x) \psi_{g}(y) \phi_{g}(z) \text {, } \\
& \boldsymbol{\Psi}_{3}^{o}(x, y, z)=\psi_{h}(x) \phi_{h}(y) \phi_{h}(z)+e_{1} \psi_{h}(x) \phi_{h}(y) \phi_{g}(z)+ \\
& +e_{2} \psi_{h}(x) \phi_{g}(y) \phi_{h}(z)+e_{3} \psi_{h}(x) \phi_{g}(y) \phi_{g}(z)+e_{4} \psi_{g}(x) \phi_{h}(y) \phi_{h}(z)+ \\
& +e_{5} \psi_{g}(x) \phi_{h}(y) \phi_{g}(z)+e_{6} \psi_{g}(x) \phi_{g}(y) \phi_{h}(z)+e_{7} \psi_{g}(x) \phi_{g}(y) \phi_{g}(z) \text {, } \\
& \boldsymbol{\Psi}_{4}^{o}(x, y, z)=\psi_{h}(x) \psi_{h}(y) \phi_{h}(z)+e_{1} \psi_{h}(x) \psi_{h}(y) \phi_{g}(z)+ \\
& +e_{2} \psi_{h}(x) \psi_{g}(y) \phi_{h}(z)+e_{3} \psi_{h}(x) \psi_{g}(y) \phi_{g}(z)+e_{4} \psi_{g}(x) \psi_{h}(y) \phi_{h}(z)+ \\
& +e_{5} \psi_{g}(x) \psi_{h}(y) \phi_{g}(z)+e_{6} \psi_{g}(x) \psi_{g}(y) \phi_{h}(z)+e_{7} \psi_{g}(x) \psi_{g}(y) \phi_{g}(z) \text {, } \\
& \boldsymbol{\Psi}_{5}^{o}(x, y, z)=\psi_{h}(x) \phi_{h}(y) \psi_{h}(z)+e_{1} \psi_{h}(x) \phi_{h}(y) \psi_{g}(z)+ \\
& +e_{2} \psi_{h}(x) \phi_{g}(y) \psi_{h}(z)+e_{3} \psi_{h}(x) \phi_{g}(y) \psi_{g}(z)+e_{4} \psi_{g}(x) \phi_{h}(y) \psi_{h}(z)+ \\
& +e_{5} \psi_{g}(x) \phi_{h}(y) \psi_{g}(z)+e_{6} \psi_{g}(x) \phi_{g}(y) \psi_{h}(z)+e_{7} \psi_{g}(x) \phi_{g}(y) \psi_{g}(z) \text {, } \\
& \boldsymbol{\Psi}_{6}^{o}(x, y, z)=\phi_{h}(x) \psi_{h}(y) \psi_{h}(z)+e_{1} \phi_{h}(x) \psi_{h}(y) \psi_{g}(z)+ \\
& +e_{2} \phi_{h}(x) \psi_{g}(y) \psi_{h}(z)+e_{3} \phi_{h}(x) \psi_{g}(y) \psi_{g}(z)+e_{4} \phi_{g}(x) \psi_{h}(y) \psi_{h}(z)+ \\
& +e_{5} \phi_{g}(x) \psi_{h}(y) \psi_{g}(z)+e_{6} \phi_{g}(x) \psi_{g}(y) \psi_{h}(z)+e_{7} \phi_{g}(x) \psi_{g}(y) \psi_{g}(z), \\
& \boldsymbol{\Psi}_{7}^{o}(x, y, z)=\psi_{h}(x) \psi_{h}(y) \psi_{h}(z)+e_{1} \psi_{h}(x) \psi_{h}(y) \psi_{g}(z)+ \\
& +e_{2} \psi_{h}(x) \psi_{g}(y) \psi_{h}(z)+e_{3} \psi_{h}(x) \psi_{g}(y) \psi_{g}(z)+e_{4} \psi_{g}(x) \psi_{h}(y) \psi_{h}(z)+ \\
& +e_{5} \psi_{g}(x) \psi_{h}(y) \psi_{g}(z)+e_{6} \psi_{g}(x) \psi_{g}(y) \psi_{h}(z)+e_{7} \psi_{g}(x) \psi_{g}(y) \psi_{g}(z) \text {. }
\end{aligned}
$$


It could be noticed that $h^{o} h^{o} h^{o}, h^{o} h^{o} g^{o}, h^{o} g^{o} h^{o}, h^{o} g^{o} g^{o}, g^{o} h^{o} h^{o}, g^{o} h^{o} g^{o}$, $g^{o} g^{o} h^{o}$ and $g^{o} g^{o} g^{o}$ are eight possible combinations of octonion filters.

Similarly to the complex and quaternionic analogues OWT produces iterative octonionic sequences as follows:

$$
\left\{A_{i-1}^{o}\right\} \rightarrow\left\{A_{i}^{o}, D_{i, j}^{o}, j=1, \ldots, 7\right\}
$$

i.e. the approximation set from the level $i-1$ to level $i$ corresponds to the transforming of one octonionic approximation set to a new quaternionic approximation set lowered eight times with respect to the previous one due to the downsampling operations.

\section{Conclusions}

The octonion wavelet transform presented in this paper extends Mallat's multiresolution algorithm by introducing the octonion wavelets. Their hypercomplex nature allows one to reach the shift-invariance and directional selectivity, which is not possible during the application of classical 3D discrete wavelet transform. The presented transform allows to improve the analysis of 3D data structures, such as magnetic resonance imaging applied in the medicine or computer tomography used for the material characterization and non-destructive testing.

\section{Acknowledgements}

The research project was financed by the National Science Centre (Poland) granted according to the decision No. DEC-2011/03/N/ST8/06205.

\section{References}

[1] Kingsbury N.G., Complex wavelets for shift invariant analysis and filtering of signals, App. Comput. Harmon. A. 2001, 10, 234-253.

[2] Liu Y., Jin J., Wang Q., Shen Y., Phases measure of image sharpness based on quaternion wavelet, Pattern Recogn. Lett. 2013, 34, 1063-1070.

[3] Lina J.-M., Complex Daubechies wavelets: filters design and applications, Proc. ISAAC Conference, University of Delaware, Newark 1997.

[4] Forster B., Blu T., Unser M., Complex B-splines and wavelets, Proc. $2^{\text {nd }}$ International Conference on Computational Harmonic Analysis, Nashville 2004.

[5] Magarey J.F.A., Kingsbury N.G., Motion estimation using a complex-valued wavelet transform, IEEE Trans. Image Process. 1998, 6, 549-565.

[6] Forster B., Blu T., Unser M., A new family of complex rotation-covariant multiresolution bases in 2D, Proc. of SPIE Conference on Mathematical Imaging: Wavelet Applications in Signal and Image Processing X, San Diego 2003, 5207, 475-479. 
[7] Bayro-Corrochano E., de La Torre Gomora M.A., Image processing using the quaternion wavelet transform, Progress in Pattern Recognition, Image Analysis and Applications, Lect. Notes Comput. Sc. 2004, 3287, 613-620.

[8] Bayro-Corrochano E., The theory and use of the quaternion wavelet transform, J. Math. Imaging Vis. 2006, 24, 19-35.

[9] Bahri M., Ashino R., Vaillancourt R., Two-dimensional quaternion wavelet transform, Appl. Math. Comput. 2011, 218, 10-21.

[10] Soulard R., Carré P., Quaternionic wavelets for texture classification, Pattern Recogn. Lett. 2011, 32, 1669-1678.

[11] Gai S., Yang G., Zhang S., Multiscale texture classification using reduced quaternion wavelet transform, Int. J. Electron. Commun. 2013, 67, 233-241.

[12] Gai S., Yang G., Wan M., Employing quaternion wavelet transform for banknote classification, Neurocomputing 2013, 118, 171-178.

[13] Conway J.H., Smith D.A., On Quaternions and Octonions: Their Geometry, Arithmetic and Symmetry, Peters, 2003.

[14] Boya L.J., Composition algebras and the two faces of $G_{2}$, arXiv:0911.3387v1, 2009.

[15] Hahn S.L., Snopek K.M., The unified theory of n-dimensional complex and hypercomplex analytic signals, Bull. Pol. Acad. Sci.-Te. 2011, 59, 167-181. 